\title{
S-CDCA: a semi-cluster directive-congestion protocol for priority-based data in WSNs
}

\author{
Marwan Ihsan Shukur \\ Department of Medical Instrumentation Technologies, Al Hikma University College (HIUC), Iraq
}

\begin{tabular}{l} 
Article Info \\
\hline Article history: \\
Received Mar 14, 2021 \\
Revised Jun 6, 2021 \\
Accepted Jun 14, 2021 \\
\hline
\end{tabular}

\section{Keywords:}

Congestion

Internet of things

Wireless networks

Wireless sensor networks

\begin{abstract}
The internet of things (IoT) protocols and regulations are being developed for various applications includes: habitat monitoring, machinery control, general health-care, smart-homes and more. A great part of I0T comprised of sensors nodes in connected networks (i.e. sensor networks.). A sensor network is a group of nodes with sensory module and computational elements connected through network interfaces. The most interesting type of sensor networks are wireless sensor networks. The nodes here are connected through wirless interfaces. The shared medium between these nodes, creates different challenges. Congestion in such network is ineavitable. Different models and methods were proposed to alleviate congestion in wireless sensor networks. This paper presents a semi-cluster directive congestion method that allivate network congestion for priority-based data transmission. The methods improve the network performance by implementing temporary cluster for low level priority data packets while providing a clear link between high priority data source node and the network base station. Simulation results show that. The proposed method outperformes ad hoc on-demand distance vector (AODV) reactive procotol approach and priority-based congestion control dynamic clustering (PCCDC) a cluster-based method in network energy consumption and control packets overhead during network operation. The proposed method also shows comparative improvments in end-to-end delays versus PCCDC.
\end{abstract}

This is an open access article under the CC BY-SA license.

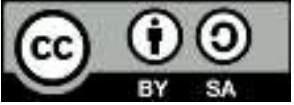

\section{Corresponding Author:}

Marwan Ihsan Shukur

Department of Medical instrumentation techniques

Al Hikma university college

Four Streets, Yarmook, Baghdad 00964, Iraq

Email: Marwan.Al-Jemeli@hiuc.edu.iq

\section{INTRODUCTION}

Wireless sensor networks (WSNs) are a promising solution that has been utilized in a variety of implementations [1], however due to dynamicity, heterogeneity, resource availability, and other factors, determining the best path is more difficult. In contrast to other areas of WSNs, residual energy of sensors in the sink coverage area is drained easily [2]. Sensor nodes are equipped with various type of sensors for different applications [3], [4].Due to the broad spectrum of current and real-world applications [5] in fields such as health sector, outer space, machinery control applications, traffic management, smart house applications, and object detection, wireless sensor networks (WSNs) has been utilized profoundly. A conventional WSN consists of a few sensor nodes dispersed across an area of interest. The area of interest can be of inaccessible environment for maintanace of the sensor nodes [2]. The sensor nodes in an application gather data from the atmosphere and send it to a data connection using single-hop or multi-hop 
communication methods [6]. Sensor nodes are operated by batteries, which are normally difficult to recharge or repair in harsh environments [7]. This is the primary limitation in developing and implementing largescale sensor networks with small energy nodes [4]. These nodes must fulfill the specifications of the particular application, such as being lightweight, inexpensive, and energy efficient, as well as providing the necessary sensors to compute and transmit the event to the base station.

Before WSN can be used in a realistic manner, certain technological problems must be solved [8]-[10]. As a consequence, network architecture must be changed to satisfy the special specifications of sensor nodes. Clustering is a useful approach to manage resources in a WSN [11], [12]. Different startiges have been implemented with focus on clusters postions and election process [13]-[15]. Motivated by the current state of the art in the field of WSNs and clustering routing approachs, this paper proposes a semi-cluster directive congestion alleviation (SCDCA) protocol. The method utilizes clustering as part of its operation to alleviate congestion by utilizing node(s) location from base station and electing specific nodes as temporary base stations.

The aim of the proposed approach is to improve the packet delivery delays of nodes with high priority information over the nodes with lower priorities. The rest of the paper is presented as follow: section 2 Illiustrate the literature and the related works that are close to goal of this paper. Section 3 describe the proposed method operation and algorithm. Section 4 describe the simulation software a parameter used for performance measurements. Section 5 discusses the results presented in this paper and finaly the conclusions.

\section{LITERATURE REVIEW AND RELATED WORK}

Cluster-based routing protocols for WSNs has been proposed in the literature [5], [7], [9], [13]. Heinzelman et al. [16] suggested the low-energy adaptive clustering hierarchy (LEACH) protocol, is one of the first implementations of clustering routing protocols in WSNs. the protocol has been used by several subsequent clustering and routing protocols. LEACH's key concept is to rotate cluster heads, distributing the heavy energy consumption for interacting with the BS across all nodes [17]. Another example of cluster-head based protocols is the hybrid energy-efficient distributed clustering (HEED) protocol, a multi-hop clustering protocol that focuses on networks energy-efficiency. Unlike the LEACH protocol, which chooses $\mathrm{CHs}$ at random, the HEED protocol chooses cluster-nodes based on energy residue in the node and the cost of the connection in terms of transmission power between the nodes [18].

Congestion allivation and load balancing [19]-[22] approaches in WSNs has been proposed through the literature. M. E. Bayrakdar [23] proposed a priority based data transmission links for WSNs for IoT applications through the use of unlicensed connection frequencies to allivate the congestion during network operation. The method requires the nodes to be equipped with specific network interfaces that are calibrated to work on unllicesnced frequnecies. The authors in [24] proposed CATopology, a congestion avoidance approach for WSNs. The approach utilizes karnough maps to build congestion trees CATree to ensure that the links in the route are congestion free through multi-hops. The authors of the study in [25] introduced the dynamic alternative path selection method a lightweight congestion management and avoidance scheme (DAlPaS). DAlPaS is an easy but efficient scheme for controlling congestion while minimizing overhead. The function of this scheme is based on resource management rather than sending rate control at the source. C. Ma et al. [26] discuss the network congestion in traffic between parent nodes and child nodes in WSNs. To alleviate the effects of network congestion, devised a game theory-based parent-shift procedure that allows child nodes to change their neighbors' nodes in the route created towards the sink node.

Priority based operation for IOT applications is of importance in the field of congestion avoidance WSNs [27]. The researchers in [7] introduced "priority based congestion control dynamic clustering (PCCDC) protocol", the main goal of the protocol is allivate network congestion whilst maintaining a competent network life-time. The protocol utilizes mobile cluster nodes that are chosen dynamically by a time slotted queue. The cluster nodes are mobile in the network. Therefore, the approach might increase control packet over-head because the network topology changes based on the node(s) mobility [8]. reliable cluster-based energy-aware routing (RCER) proposed by the researchers in [28] is another example of cluster-based routing in WSNs with focus on increasing network-lifetime whilst maintaining optimum roundtime-trip (RTT) for packet delivery. The interesting point of the porposed approach is targeting heterogenous sensor nodes. The main steps of the protocol are: devide the deployment area into sections for the clusters, implementing a route section metric that is based on a node(s) residual energy and link RTT. Table 1 summarizes the related works discussed in this paper. From the related work section, the porposed method will be compared against PCCDC protocol as it focuses on priority link operation and its cluster-head selection operation mechanism. 
Table 1. Related works summary

\begin{tabular}{llcc}
\hline \multicolumn{1}{c}{ Protocol } & \multicolumn{1}{c}{ Method of operation } & Congestion control & Priority data \\
\hline LEACH & Cluster-based & No & No \\
HEED & Cluster-based & No & No \\
[24] M.E. Barakdar & Licenced and Unlicenced operation spectrum & Yes & Yes \\
CATopology [25] & Ad-hoc operation & Yes & No \\
DAlPaS [26] & Ad-hoc operation with resource management control & Yes & No \\
Game theory-based parent- & Clustering with game-theory approach & Yes & Yes \\
shift procedure [27] & & No & No \\
PCCDC [7] & Clustering & & Clustering with heterogenous nodes \\
RCER [28] & & &
\end{tabular}

\section{PROPOSED METHOD}

The proposed method in this paper utilizes the concept of creating temporary clusters to direct nodes data towards them (Figure 1). There are two tiers of data in the network, high-priority (HP-data) and lowpriority (LP-data). The network starts its operation by populating control packets, node info. packet (NIP) in a proactive manner. NIP packet includes the actual location of the node. The location information is used to calculate the distance between the source node and the neighboring nodes. This operation creates a link-list for the neighoring nodes with thier actual location. The NIP packets are populated until the nodes have fully created lists of the neighboring nodes.

The nodes then starting route discovery for the base-station of the network. In the event of node having to send a HP-data, the source node sends a short message to the network requesting a clear route to the base station (Figure 2). The base station starts electing temporary cluster nodes in the network as a location for the other nodes with LP-data to send their data packets to. By isolating the network broadcast to clusters, the network congestion alliveated and the node with the HP-data have a clear route to the base station.

The base-station chooses the temporary cluster nodes from the nodes in the network. The temporary cluster nodes are chosen based on HP-data source node location. The base station measures its distance from the source node and chooses the cluster nodes by adding half the distance (or subtracting depending on the location of the source node) to the X-axis of the source node location for the first temporary cluster, and the same value is added (or subtracted depending on the source node location) to the y-axis of the source node (Figure 3).

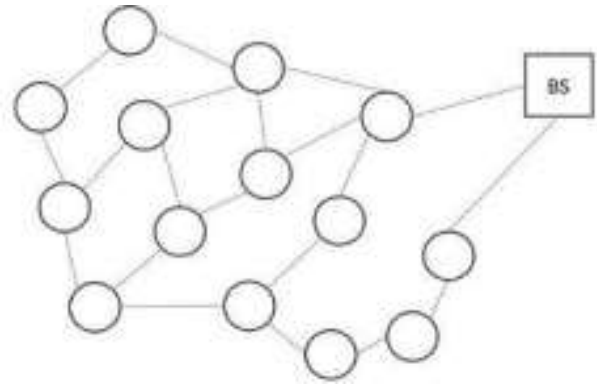

Figure 1. Network example

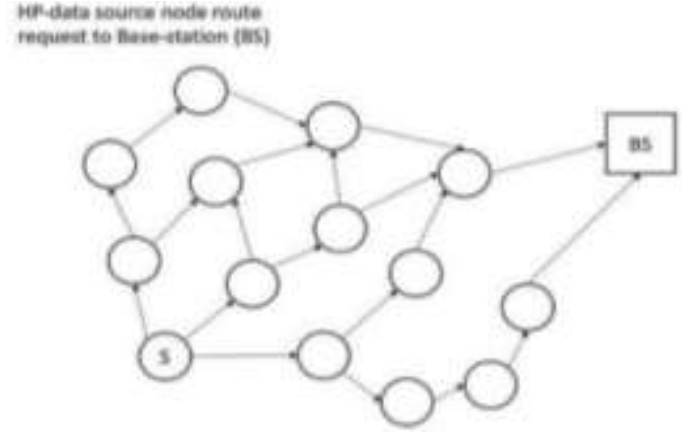

Figure 2. HP-Data source node request link to basestation packet delivery process

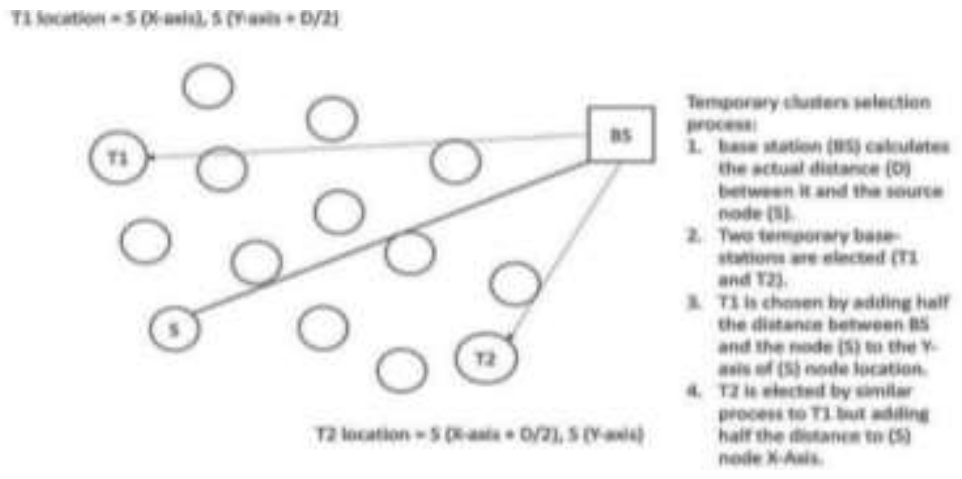

Figure 3. Temporary clusters selection process 
The source node then chooses the route with shortest path to the base-station. The temporary cluster nodes improve the network congestion by promoting themselves as temporary base-station for the nodes with LP-data that are close in location to HP-data source node (Figure 4).
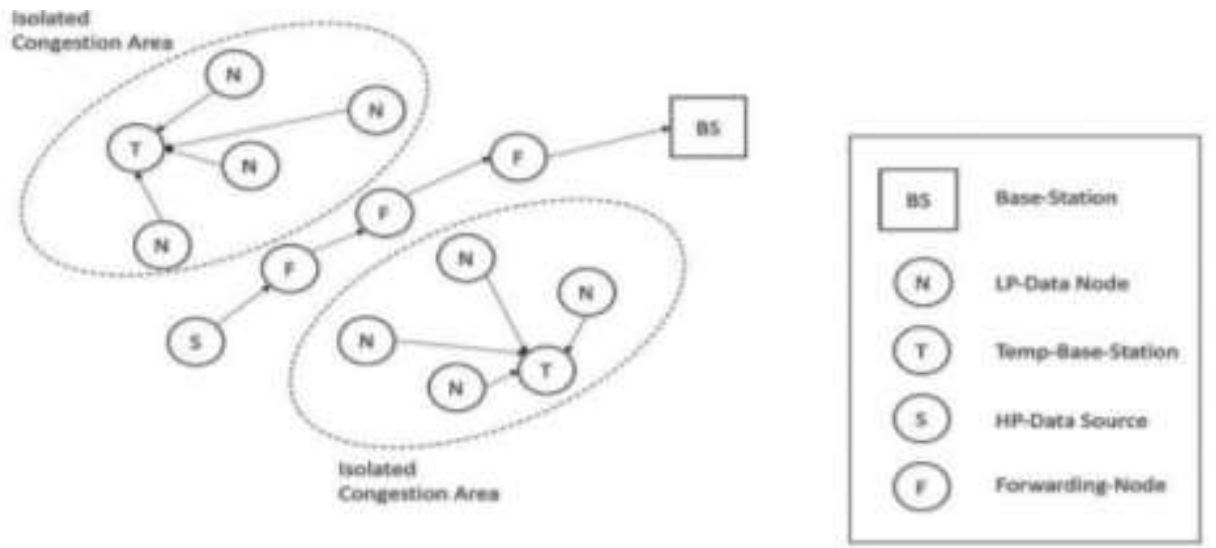

Figure 4. Network virtual layout after setup process

\section{SIMULATION SETUP AND PARAMETERS}

The Network simulator 2 [29] (NS2 version 2.34) software was used as the software to perform measurments analysis and simulations. The simulations were perfomed over (11) network scenarios in a depolyment area of $(1000 \times 1000)$ square meters. Figure 5 below illustrate an example of a network deployment. The proposed method has been compared against AODV protocol [30] to represent a reactive on-demand protocol approach. And compared against PCCDC [7] method to represent protocols with clusters (i.e. LEACH protocol and derevatives). However, PCCDC was also chosen because it promotes priority data transimission to base-station. Table 2 illustrate the simulations parameters used for measuring the performance of the proposed method over the other methods. Every simulation run was repeated for (31) times to achieve a confidence interval of $(95 \%)$ for the collected results

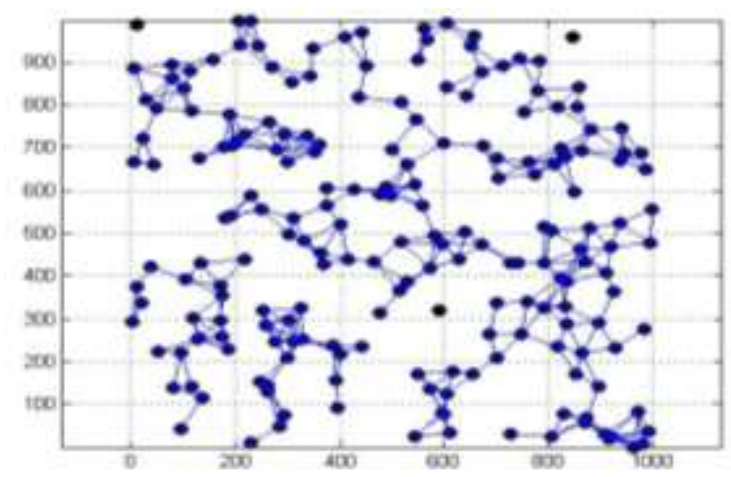

Figure 5. Example of sensor nodes deployment layout for (130) nodes

Table 2. Simulation parameters

\begin{tabular}{ll}
\hline \multicolumn{1}{c}{ Parameter } & \multicolumn{1}{c}{ Value } \\
\hline Number of nodes for each simulation scenario & $30,50,70,90,110,130,150,170,190,210$ \\
Deployment area & $1000 \times 1000$ square meters \\
Number of source nodes in each simulation & 5 \\
Transimission range of the node & 30 meters \\
Reception rang of the node & 60 meters \\
Nodes initial energy & 1000 joules \\
Propagation model & Two-Ray-Ground \\
Simulation period & 1000 seconds \\
Operation frequency & $2.4 \mathrm{GHz}$ \\
Data transmission rate & $250 \mathrm{Kbs}$ \\
\hline
\end{tabular}

S-CDCA: a semi-cluster directive-congestion protocol for priority-based data in...(Marwan Ihsan Shukur) 


\section{RESULTS AND DISCUSSION}

This section illustrates the results of the performed simulation. Section 5.1 discusses the network energy consumption performance. Section 5.2 discusses the network overall packets overhead. Finally, section 5.3 discusses the proposed method performance in terms of packet delivery end-to-end delays.

\subsection{Network energy consumption results}

Figure 6 illustrate the network average energy consumption for the proposed method versus AODV reactive protocol and Cluster-head operation protocol. From the figure it shows that the directive-congestion approach performce better than AODV operation and the Cluster-head methods. The method fares better against cluster-based algorithm. AODV energy is due to the high number of generated control packets creating an increase in the energy utilized by the nodes to keep the links from the source to the destination available for the source nodes. Cluster-head algorithm performs better than AODV largly because of the decrease in the generated control packets by the network to maintain the links created between the nodes. However, the directive-congestion method performs better than the cluster-head method because of the decrease in the generated control packets.

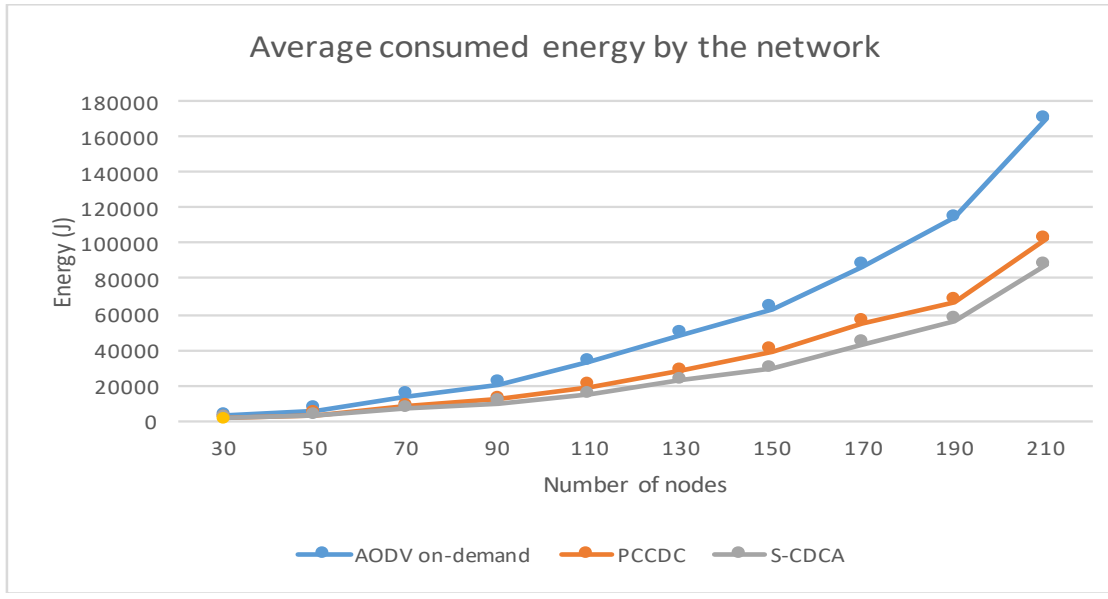

Figure 6. Average energy consumed by the network

\subsection{Control packets overhead results}

The generation of control packets in a network increases network congestion. Over-hearing in the network also increases the chances of congestion in the network. Figure 7 shows the performance of the directive-congestion method in terms of control packets generation.

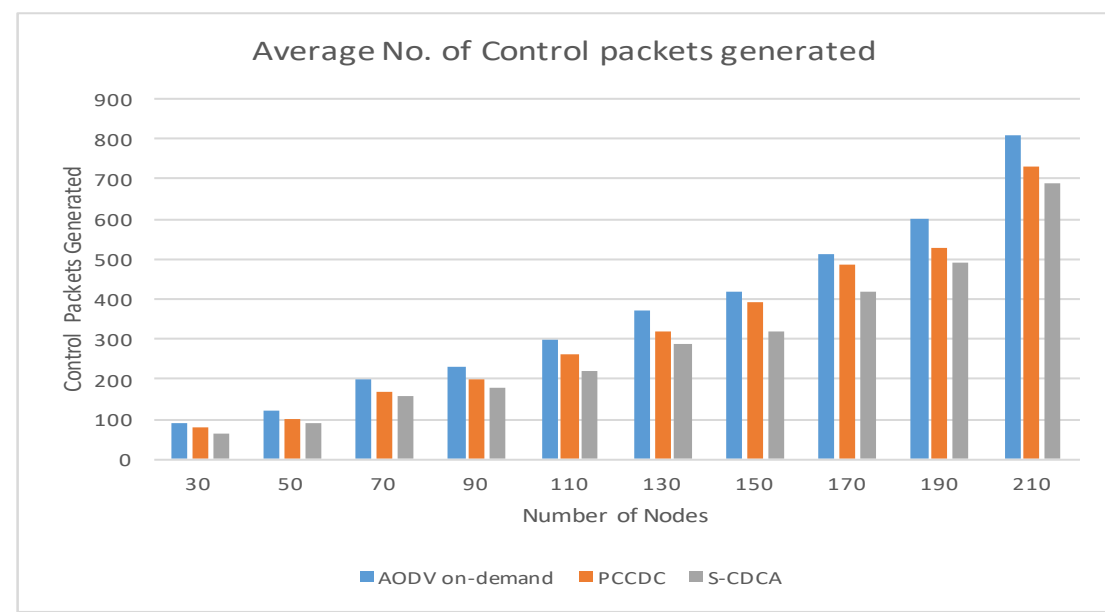

Figure 7. Control packets generated by the network 


\subsection{End-to-End delay results}

Controlling the number of packets generated in the network improves the packet delivery delays from source to destination. Figure 8 illustrate the end-to-end delays results. From the figure, increasing the number of nodes in the network increases the end-to-end delay of the packet. This is due to the increase in general in the numbr of hops for the created routes from source to base-station. AODV operation requires although on-demand the routes are created, the propagation of the route discovery packets increases the network congestion. Resulting in higher end-to-end delay in packets versus cluster-head operation and directive-congestion. Cluster-head method creates cluster-heads in a random manner. Directive-congestion control employs temporary base-station nodes rather than clusters. Those nodes are responsible for collecting LP-Data from the nodes whilst leaving the link for the HP-data source node to the destination (the Basestation) clear from consgestion created by control packets. Therefore, the proposed method performs better in terms of end-to-end delays against the other compared methods.

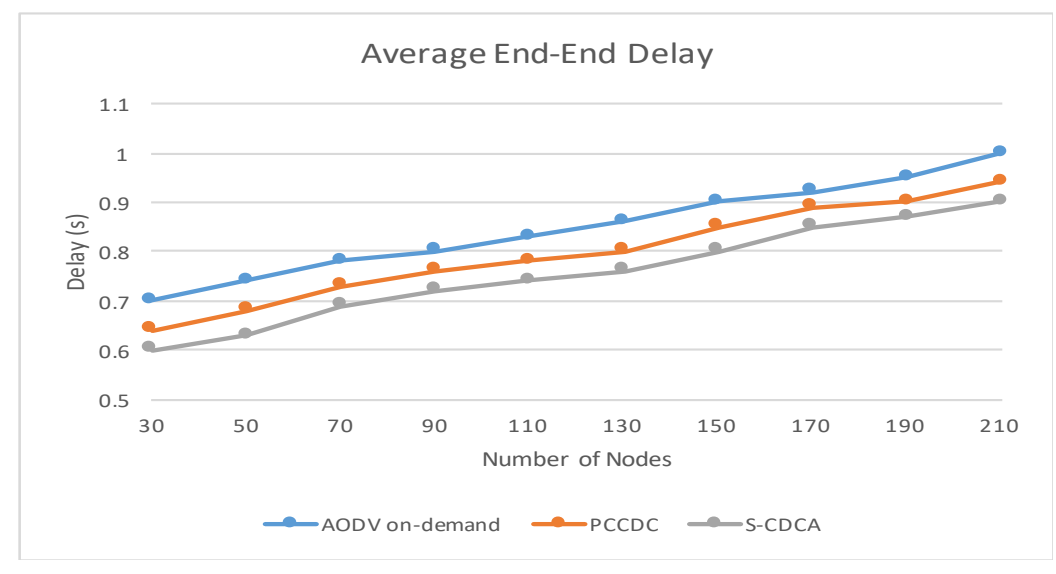

Figure 8. End-to-end packet delays

\section{CONCLUSION}

This paper has presented a priority-based directive-congestion alleviation method for WSNs. Through simulations the method has performed better than AODV reactive on-demand protocol opertation and clusterbased protocol operation in terms of network energy consumption, control packets overhead and packet delivery end-to-end delays. The proposed method can be utilized for applications were priorty of data is in consideration to achieve optimal data delivery delays. The main strength point of the proposed method is the implamentation of temporary base-station nodes for LP-data. The criteria of chosing temporary base station (BS) nodes are strike a balanced operation over the network. The proposed method operation can be improved by applying different temporary clusters selection methods. A future work for this paper is improve the proposed method to be utilized on mobile-WSNs. Mobility increases the challenegs in chosing the temporary BS-Nodes because the network topology might change rapidly based the node(s) mobility charctaristics.

\section{REFERENCES}

[1] C.-C. Lee, "Security and Privacy in Wireless Sensor Networks: Advances and Challenges," Sensors, vol. 20, no. 3, Jan. 2020, doi: 10.3390/s20030744.

[2] H. Hematkhah and Y. S. Kavian, "DCPVP: Distributed Clustering Protocol Using Voting and Priority for Wireless Sensor Networks,” Sensors, vol. 15, no. 3, 5763-5782., Mar. 2015, doi: 10.3390/s150305763.

[3] M. S. BenSaleh, R. Saida, Y. H. Kacem, and M. Abid, "Wireless Sensor Network Design Methodologies: A Survey," J. Sens., vol. 2020, p. e9592836, Jan. 2020, doi: 10.1155/2020/9592836.

[4] M. E. Bayrakdar, "Employing sensor network based opportunistic spectrum utilization for agricultural monitoring," Sustain. Comput. Inform. Syst., vol. 27, p. 100404, Sep. 2020, doi: 10.1016/j.suscom.2020.100404.

[5] K. W. Al-Ani, "Unequal Clustering in Wireless Sensor Network: A Review," Indonesian Journal of Electrical Engineering and Computer Science, vol. 22, no. 1, pp. 419-426, Apr. 2021, doi: 10.11591/ijeecs.v22.i1.pp\%p.

[6] B.-S. Kim, K.-I. Kim, B. Shah, F. Chow, and K. H. Kim, "Wireless Sensor Networks for Big Data Systems," Sensors, vol. 19, no. 7, Apr. 2019, doi: 10.3390/s19071565.

[7] R. Beulah Jayakumari and V. Jawahar Senthilkumar, "Priority Based Congestion Control Dynamic Clustering Protocol in Mobile Wireless Sensor Networks," Sci. World J., vol. 2015, p. 596138, Oct. 2015, doi: 10.1155/2015/596138. 
[8] M. Al-Jemeli and F. A. Hussin, "An Energy Efficient Cross-Layer Network Operation Model for IEEE 802.15.4-Based Mobile Wireless Sensor Networks," IEEE Sens. J., vol. 15, no. 2, pp. 684-692, Feb. 2015, doi: 10.1109/JSEN.2014.2352041.

[9] O. Deepa and J. Suguna, "An optimized QoS-based clustering with multipath routing protocol for Wireless Sensor Networks," J. King Saud Univ. - Comput. Inf. Sci., vol. 32, no. 7, pp. 763-774, 2020, doi: 10.1016/j.jksuci.2017.11.007.

[10] M. E. Bayrakdar, "Cooperative communication based access technique for sensor networks," Int. J. Electron., vol. 107, no. 2, pp. 212-225, Feb. 2020, doi: 10.1080/00207217.2019.1636313.

[11] Y. Zhang, W. Xiong, D. Han, W. Chen, and J. Wang, "Routing Algorithm with Uneven Clustering for Energy Heterogeneous Wireless Sensor Networks," J. Sens., vol. 2016, p. e7542907, Sep. 2016, doi: 10.1155/2016/7542907.

[12] B. Me, "Priority based health data monitoring with IEEE 802.11af technology in wireless medical sensor networks.," Med. Biol. Eng. Comput., vol. 57, no. 12, pp. 2757-2769, Nov. 2019, doi: 10.1007/s11517-019-02060-4.

[13] M. Ilyas et al., "Trust-based energy-efficient routing protocol for Internet of things-based sensor networks," Int. J. Distrib. Sens. Netw., vol. 16, no. 10, Oct. 2020, doi: 10.1177/1550147720964358.

[14] A. G.r. and Gowrishankar, "Energy efficient clustering and routing in a wireless sensor networks," Procedia Comput. Sci., vol. 134, pp. 178-185, Jan. 2018, doi: 10.1016/j.procs.2018.07.160.

[15] P. Kuila and P. K. Jana, "An energy balanced distributed clustering and routing algorithm for Wireless Sensor Networks," in 2012 2nd IEEE International Conference on Parallel, Distributed and Grid Computing, Dec. 2012, pp. 220-225, doi: 10.1109/PDGC.2012.6449821.

[16] W. R. Heinzelman, A. Chandrakasan, and H. Balakrishnan, "Energy-efficient communication protocol for wireless microsensor networks," in Proceedings of the 33rd Annual Hawaii International Conference on System Sciences, Jan. 2000, p. 10 pp. vol.2-, doi: 10.1109/HICSS.2000.926982.

[17] N. G. Palan, B. V. Barbadekar, and S. Patil, "Low energy adaptive clustering hierarchy (LEACH) protocol: A retrospective analysis," in 2017 International Conference on Inventive Systems and Control (ICISC), Jan. 2017, pp. 1-12, doi: 10.1109/ICISC.2017.8068715.

[18] H. Wang, Q. Liu, and M. Liu, "NHEED: An energy-efficient multi-hop routing protocol based on HEED," 2016 12th World Congress on Intelligent Control and Automation (WCICA), 2016, pp. 3219-3225, doi: 10.1109/WCICA.2016.7578409.

[19] H. Kim, H. Kim, J. Paek, and S. Bahk, "Load Balancing Under Heavy Traffic in RPL Routing Protocol for Low Power and Lossy Networks,” IEEE Trans. Mob. Comput., vol. 16, no. 4, pp. 964-979, Apr. 2017, doi: 10.1109/TMC.2016.2585107.

[20] H. -S. Kim, J. Paek, and S. Bahk, "QU-RPL: Queue utilization based RPL for load balancing in large scale industrial applications," 2015 12th Annual IEEE International Conference on Sensing, Communication, and Networking (SECON), 2015, pp. 265-273, doi: 10.1109/SAHCN.2015.7338325.

[21] H. A. A. Al-Kashoash, H. Kharrufa, Y. Al-Nidawi, and A. H. Kemp, "Congestion control in wireless sensor and 6LoWPAN networks: toward the Internet of Things," Wirel. Netw., vol. 25, no. 8, pp. 4493-4522, Nov. 2019, doi: 10.1007/s11276-018-1743-y.

[22] H. A. Kashoash, M. Hafeez, and A. Kemp, "Congestion control for 6LoWPAN networks: A game theoretic framework," IEEE Internet Things J., vol. 4, no. 3, pp. 760-771, Jun. 2017, doi: 10.1109/JOT.2017.2666269.

[23] M. E. Bayrakdar, "Exploiting cognitive wireless nodes for priority-based data communication in terrestrial sensor networks," ETRI J., vol. 42, no. 1, pp. 36-45, 2020, doi: 10.4218/etrij.2019-0296.

[24] R. Dasgupta, R. Mukherjee, and A. Gupta, "Congestion avoidance topology in wireless sensor network using Karnaugh map," in 2015 Applications and Innovations in Mobile Computing (AIMoC), Feb. 2015, pp. 89-96, doi: 10.1109/AIMOC.2015.7083835.

[25] C. Sergiou, V. Vassiliou, and A. Paphitis, "Congestion control in Wireless Sensor Networks through dynamic alternative path selection,” Comput. Netw., vol. 75, no. PA, pp. 226-238, Dec. 2014, doi: 10.1016/j.comnet.2014.10.007.

[26] C. Ma, J.-P. Sheu, and C.-X. Hsu, "A Game Theory Based Congestion Control Protocol for Wireless Personal Area Networks,” J. Sens., vol. 2016, p. e6168535, Dec. 2015, doi: 10.1155/2016/6168535.

[27] F. Safara, A. Souri, T. Baker, I. Al Ridhawi, and M. Aloqaily, "PriNergy: a priority-based energy-efficient routing method for IoT systems," J. Supercomput., vol. 76, no. 11, pp. 8609-8626, Nov. 2020, doi: 10.1007/s11227-020-03147-8.

[28] K. Haseeb et al., "RCER: Reliable Cluster-based Energy-aware Routing protocol for heterogeneous Wireless Sensor Networks," PLOS ONE, vol. 14, no. 9, Sep. 2019, doi: 10.1371/journal.pone.0222009.

[29] The Network Simulator - ns-2. [Online]. Available: https://www.isi.edu/nsnam/ns/. (Accessed Mar. 13, 2021).

[30] E. M. Royer and C. E. Perkins, An implementatioE. M. Royer and C. E. Perkins, "An implementation study of the AODV routing protocol," 2000 IEEE Wireless Communications and Networking Conference. Conference Record (Cat. No.00TH8540), 2000, pp. 1003-1008 vol.3, doi: 10.1109/WCNC.2000.904764.

\section{BIOGRAPHY OF AUTHOR}

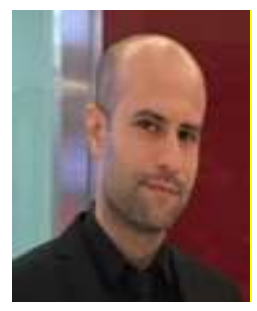

Marwan Al-Jemeli received the B.Sc. degree in computer engineering from the University of Baghdad, College of Engineering, Baghdad, Iraq, in 2005 and the M.Sc. degree in electrical and electronic engineering from Universiti Teknologi Petronas, Perak, Malaysia, in 2010. He received his Phd. Degree in electrical and electronics engineering from university Teknology PETRONAS. Since 2017 he is with Al Hikma University college, Dept. of Medical instrumentation techniques. His current research interests include computer networks, wireless network, wireless sensor networks, routing, medium access control, location estimation, and mobility in wireless networks 